\title{
Enhancement of cAMP-mediated inotropic responses by CNP is regulated differently by PDE2 in normal and failing hearts
}

\author{
Silja Meier ${ }^{1,2^{*}}$, Kjetil Wessel Andressen ${ }^{1,2}$, Jan Magnus Aronsen ${ }^{2,3,4}$, Ivar Sjaastad ${ }^{2,3}$, Tor Skomedal ${ }^{1,2}$, \\ Jan-Bjørn Osnes ${ }^{1,2}$, Eirik Qvigstad ${ }^{1,2}$, Finn Olav Levy ${ }^{1,2}$, Lise Román Moltzau ${ }^{1,2}$ \\ From 7th International Conference on cGMP Generators, Effectors and Therapeutic Implications \\ Trier, Germany. 19-21 June 2015
}

\section{Background}

Natriuretic peptide levels are increased in heart failure (HF). Atrial (ANP) and brain (BNP) natriuretic peptide mediate their effects preferentially through the natriuretic peptide receptor (NPR)-A, and C-type natriuretic peptide (CNP) through NPR-B. NPRs are membrane bound guanylyl cyclases that increase cyclic GMP (cGMP) production when activated. We have previously shown that NPR-B stimulation by CNP enhances $\beta_{1}$-adrenoceptor $\left(\beta_{1}-\mathrm{AR}\right)$ - and $5-\mathrm{HT}_{4}$ serotonin receptor-mediated signaling in failing hearts, probably through inhibition of phosphodiesterase (PDE) 3, a potential detrimental effect in the failing heart. In this study we examine the role of PDE2 in regulating the CNP-induced enhancement of $\beta_{1}-\mathrm{AR}$ and $5-\mathrm{HT}_{4}$ signaling in non-failing (Sham) and failing (HF) hearts, as PDE2 is a dual-selective PDE and can potentially be stimulated by cGMP.

\section{Methods}

Chronic heart failure was induced in male Wistar rats by 6 -week coronary artery ligation. Contractility studies were performed ex vivo in left ventricular muscle strips in the presence of appropriate receptor agonist and antagonists. cGMP measurements were performed on isolated left ventricular cardiomyocytes and PDE activity assays on left ventricular cardiomyocyte homogenates.

\section{Results}

CNP, by stimulating NPR-B, was able to enhance $\beta_{1}$-ARand $5-\mathrm{HT}_{4}$-mediated inotropic responses in Sham and $\mathrm{HF}$

\footnotetext{
* Correspondence: silja.meier@medisin.uio.no

'Department of Pharmacology, University of Oslo and Oslo University

Hospital, Oslo, Norway

Full list of author information is available at the end of the article
}

left ventricular strips. CNP elicited a similar increase of cGMP in cardiomyocytes from Sham and HF. cGMP reduced the CAMP-PDE activity of PDE3 and increased the cAMP-PDE activity of PDE2 concentration-dependently in cardiomyocyte homogenates in a similar way in Sham and HF. In Sham inhibition of PDE2 by EHNA amplified the CNP-induced enhancement of $\beta_{1}$-AR- and 5- $\mathrm{HT}_{4}$-mediated inotropic responses. In HF PDE2 inhibition did not influence the functional effects of CNP despite increasing the cGMP response to the same marked extent as in Sham.

\section{Conclusions}

There is a preserved mechanism of CNP-induced enhancement of $\beta_{1}$-AR and $5-\mathrm{HT}_{4}$ in Sham and HF. cGMP levels and cGMP-mediated activation and inhibition of cAMP-PDE activity of PDE2 and PDE3, respectively, are similar in Sham and HF. However, PDE2 seems more involved in regulating the $\beta_{1}-\mathrm{AR}$ and $5-\mathrm{HT}_{4}$ enhancement in Sham compared to HF, which might reflect differences between Sham and HF in PDE2 expression or compartmentation.

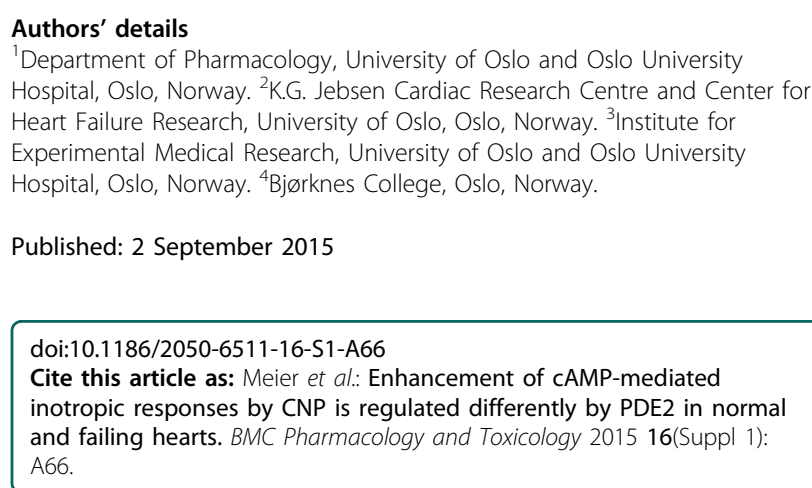
Heart Failure Research, University of Oslo, Oslo, Norway. ${ }^{3}$ Institute for Experimental Medical Research, University of Oslo and Oslo University Hospital, Oslo, Norway. ${ }^{4}$ Bjørknes College, Oslo, Norway.

Published: 2 September 2015

doi:10.1186/2050-6511-16-S1-A66

Cite this article as: Meier et al:: Enhancement of cAMP-mediated inotropic responses by CNP is regulated differently by PDE2 in normal and failing hearts. BMC Pharmacology and Toxicology 2015 16(Suppl 1): A66. 